\title{
An integrated platform for intuitive mathematical programming modeling using LaTeX
}

\author{
Charalampos P Triantafyllidis ${ }^{1,2}$, Lazaros G. Papageorgiou ${ }^{\text {Corresp. } 1}$ \\ ${ }^{1}$ Department of Chemical Engineering, University College London, University of London, London, United Kingdom \\ 2 Smith School of Enterprise and the Environment, University of Oxford, Oxford, United Kingdom \\ Corresponding Author: Lazaros G. Papageorgiou \\ Email address: I.papageorgiou@ucl.ac.uk
}

This paper presents a novel prototype platform that uses the same LaTeX mark-up language, commonly used to typeset mathematical content, as an input language for modeling optimization problems of various classes. The platform converts the LaTeX model into a formal Algebraic Modeling Language (AML) representation based on Pyomo through a parsing engine written in Python and solves by either via NEOS server or locally installed solvers, using a friendly Graphical User Interface (GUI). The distinct advantages of our approach can be summarized in i) simplification and speed-up of the model design and development process ii) non-commercial character iii) cross-platform support iv) easier typo and logic error detection in the description of the models and v) minimization of working knowledge of programming and AMLs to perform mathematical programming modeling. Overall, this is a presentation of a complete workable scheme on using LaTeX for mathematical programming modeling which assists in furthering our ability to reproduce and replicate scientific work. 
1 An integrated platform for intuitive mathematical programming

2

4

5

6

7 modeling using $\mathrm{AT}_{\mathrm{E} X}$

\author{
Charalampos P. Triantafyllidis ${ }^{\mathrm{a}, \mathrm{b}}$, Lazaros G. Papageorgiou ${ }^{\mathrm{a}, *}$ \\ ${ }^{a}$ Centre for Process Systems Engineering, Department of Chemical Engineering, \\ UCL (University College London), UK \\ ${ }^{b}$ Smith School of Enterprise and the Environment, \\ University of Oxford, UK
}

\title{
8 Abstract
}

9 This paper presents a novel prototype platform that uses the same $\mathrm{FT}_{\mathrm{E} X} \mathrm{mark}$-up language,

10 commonly used to typeset mathematical content, as an input language for modeling opti11 mization problems of various classes. The platform converts the $\mathrm{AT}_{\mathrm{E}} \mathrm{X}$ model into a formal 12 Algebraic Modeling Language (AML) representation based on Pyomo through a parsing en13 gine written in Python and solves by either via NEOS server or locally installed solvers, 14 using a friendly Graphical User Interface (GUI). The distinct advantages of our approach 15 can be summarized in i) simplification and speed-up of the model design and development 16 process ii) non-commercial character iii) cross-platform support iv) easier typo and logic er17 ror detection in the description of the models and v) minimization of working knowledge of 18 programming and AMLs to perform mathematical programming modeling. Overall, this is a 19 presentation of a complete workable scheme on using $\mathrm{LT}_{\mathrm{E} X} \mathrm{X}$ for mathematical programming modeling which assists in furthering our ability to reproduce and replicate scientific work.

20 Keywords: LATEX, Python, Pyomo, Algebraic Modeling Languages, Mathematical

21 Programming; Optimization;

222010 MSC: 90C05, 90C11, 90C90, 97M10, 68T35, 97P30

\footnotetext{
*Corresponding Author

Email addresses:

h.triantafyllidis@ucl.ac.uk, charalampos.triantafyllidis@smithschool.ox.ac.uk (Charalampos P. Triantafyllidis ), 1.papageorgiou@ucl.ac.uk (Lazaros G. Papageorgiou )
} 


\section{1. Introduction}

Mathematical modeling constitutes a rigorous way of inexpensively simulating complex systems' behavior in order to gain further understanding about the underlying mechanisms and trade-offs. By exploiting mathematical modeling techniques, one may manipulate the system under analysis so as to guarantee its optimal and robust operation.

The dominant computing tool to assist in modeling is the Algebraic Modeling Languages (AMLs) (Kallrath, 2004). AMLs have been very successful in enabling a transparent development of different types of models, easily distributable among peers and described with clarity, effectiveness and precision. Software suites such as AIMMS (Bisschop and Roelofs, 2011), GAMS IDE (Bruce A. McCarl et. al., 2013), JuMP (Dunning et al., 2017) as the modeling library in Julia (Lubin and Dunning, 2015), Pyomo ${ }^{1}$ (Hart et al., 2017, 2011) for modeling in Python ${ }^{2}$, (Rossum, 1995) and AMPL (Fourer et al., 1993) are the most popular and widely used in both academia and industry. AMLs usually incorporate the following features:

- a strict and specific syntax for the mathematical notation to describe the models;

- Solver interfaces, the bridge between mathematics and what the solver can understand in terms of structural demands;

- a series of available optimization solvers for as many classes of problems as supported (LP, MILP, MINLP etc.) with the associated functional interfaces implemented;

- explicit data file formats and implementation of the respective import/export mechanisms.

AMLs provide a level of abstraction, which is higher than the direct approach of generating a model using directly a programming language. The different levels in the design process of a model are depicted in Figure 1. Extending an AML (or even the entire modeling design process) can be done in the following two ways: we can either simplify the present framework

\footnotetext{
${ }^{1}$ http://www.Pyomo.org/

${ }^{2}$ https://www.python.org/
} 
48 (vertical abstraction) or extend the embedded functionality (horizontal abstraction) (Jackson, 49 2012). The layers of abstraction between the conception and the semantics of a mathematical 50 model and its computational implementation may not necessarily be thin. This means that 51 while eventually the aim of the presented platform has the same purpose as an AML that 52 is to generate and solve models, simplification of the required syntax to describe the model 53 is associated with higher complexity. Thus, in order to relax the syntactical requirements, 54 we have to be able to process the same model with limited information (for instance, we do 55 not declare index sets and parameters in the platform). This limited declaration of model 56 components elevates the amount of processing that the platform has to conduct in order to 57 provide equivalent formulations of the input.

A systems approach, MOSAIC (Erik et al.), has been developed based on a MathML representation using $\mathrm{LT}_{\mathrm{E}} \mathrm{X}$ extracts, which has been applied mainly to chemical engineering models. Both frameworks can be facilitated online, with the proposed framework built on Django while MOSAIC on Java. It can be noted that our platform can also be run offline (locally). A key difference between the two is that in the proposed framework the user does not explicitly define indices, parameters and dynamic sets as those are identified automatically from the platform, by filtering them out from the variable list given at the bottom of the input .tex model. In the proposed platform the user can capture the entire optimization model in a single .tex file and use this directly as an input to the platform as opposed of using ATEX extracts for generating equations in MOSAIC. Similarly though, both platforms are framing the use of $\mathrm{AT}_{\mathrm{E} X}$ built-in commands for the specific environment to better capture errors and provide more consistency. Finally, the proposed platform exports the generated optimization model in Pyomo whereas the ability to export in many other formats is given in the MOSAIC environment.

Our work expands upon two axes : i) the programming paradigm introduced by Donald E. Knuth (Knuth, 1984) on Literate Programming and ii) the notions of reproducible and replicable research, the fundamental basis of scientific analysis. Literate Programming focuses 5 on generating programs based on logical flow and thinking rather than being limited by the imposing syntactical constraints of a programming language. In essence, we employ a simple mark-up language, $\mathrm{AT}_{\mathrm{E}} \mathrm{X}$, to describe a problem (mathematical programming model) and 


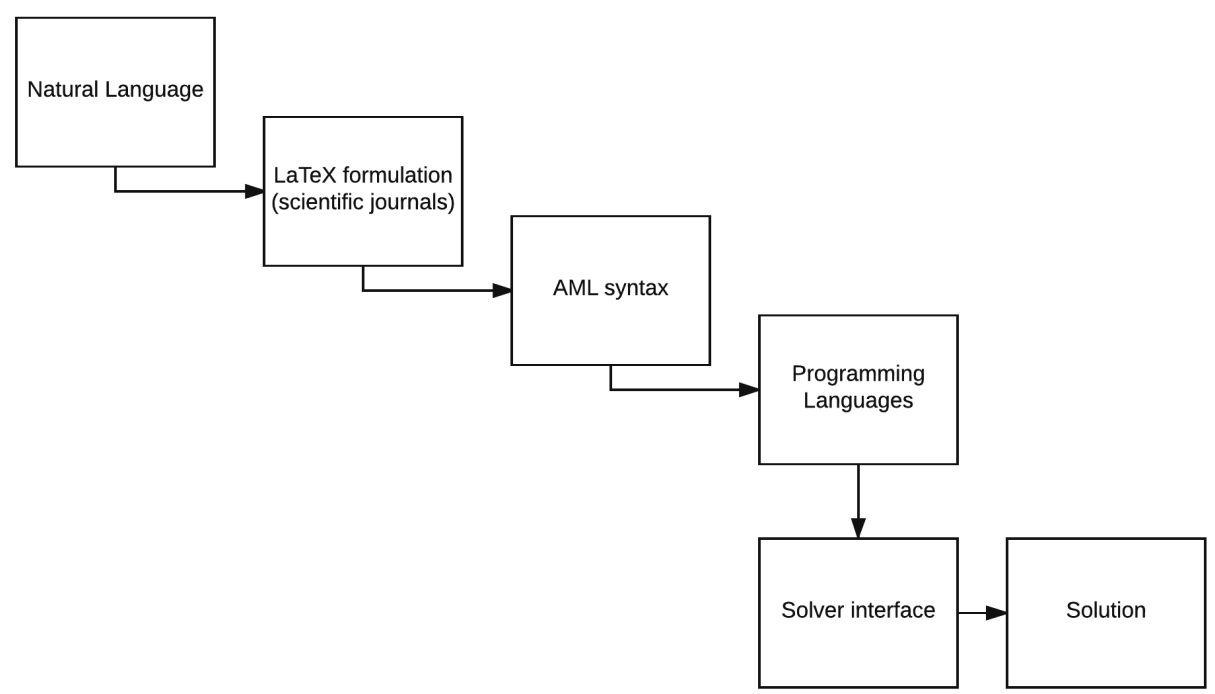

Figure 1: The levels of abstraction in modeling; from natural language to extracting the optimal solution via computational resources.

78 then in turn produce compilable code (Pyomo abstract model) which can be used outside of 79 the presented prototype platform's framework. Reproducibility and the ability to replicate 80 scientific analysis is crucial and challenging to achieve. As software tools become the vessel to 81 unravel the computational complexity of decision-making, developing open-source software 82 is not necessarily sufficient; the ability for the averagely versed developer to reproduce and 83 replicate scientific work is very important to effectively deliver impact (Leek and Peng, 2015; 84 Editorial, 2014). To quote the COIN-OR Foundation ${ }^{3}$, Science evolves when previous results 85 can be easily replicated.

In the endeavor of simplifying the syntactical requirements imposed by AMLs we have developed a prototype platform. This new framework is materializing a level of modeling design that is higher than the AMLs in terms of vertical abstraction. It therefore strengthens the ability to reproduce and replicate optimization models across literature for further anal90 ysis by reducing the demands in working knowledge of AMLs or coding. The key capability 91 is that it parses $\mathrm{H}_{\mathrm{E}} \mathrm{E}$ X formulations of mathematical programs (optimization problems) di2 rectly into Pyomo abstract models. The framework then combines the produced abstract 3 model with data provided in the AMPL .dat format (containing parameters and sets) to

\footnotetext{
${ }^{3}$ https://www.coin-or.org/
} 
94 produce a concrete model. This capability is provided through a graphical interface which 95 accepts $\mathrm{AT}_{\mathrm{E} X}$ input and AMPL data files, parses a Pyomo model, solves with a selected 96 solver (CPLEX, GLPK, or the NEOS server), and returns the optimal solution if feasible, 97 as the output. The aim is not to substitute but to establish a link between those using a 98 higher level of abstraction. Therefore, the platform does not eliminate the use of an AML 99 or the advantages emanating from it.

100 This is a complete prototype workable scheme to address how LATEX could be used as 101 an input language to perform mathematical programming modeling, and currently supports 102 Linear Programming (LP), Mixed-Integer Linear Programming (MILP) as well as Mixed103 Integer Quadratic Programming (MIQP) formulations. Linear Optimization (Bertsimas and 104 Tsitsiklis, 1997; Williams, 1999) has proven to be an invaluable tool for decision support 105 over the past decades. The corpus of models invented for linear optimization over the past 106 decades and for a multitude of domains has been consistently increasing. It can be easily 107 demonstrated with examples in Machine Learning, Operations Research and Management 108 Science, Physics, Information Security, Environmental Modeling and Systems Biology among 109 many others (Yang et al., 2016; Tanveer, 2015; Silva et al., 2016; Sitek and Wikarek, 2015; 110 Liu and Papageorgiou, 2018; Triantafyllidis et al., 2018; Cohen et al., 2017; Romeijn et al., 111 2006; Mitsos et al., 2009; Melas et al., 2013; Kratica et al., 2014; Mouha et al., 2012).

113 ported by the platform at this prototype stage. In section 3, we present the implementation 114 details of the parser. Section 4 provides a description of an illustrative example. A discussion 115 follows in section 5. Some concluding remarks are drawn in section 6. Examples of opti116 mization models that were reproduced from scientific papers as well as their corresponding 117 LTEX formulations and Pyomo models can be found in the Supplementary Information.

\section{2. Functionality}

119 The set of rules that are admissible to formulate models in this platform are formal $\mathrm{LT}_{\mathrm{E}} \mathrm{X}$ 120 commands and they do not represent in-house modifications. We assume that the model will 121 be in the typical format that optimization programs commonly appear in scientific journals.

122 Therefore, the model must contain the following three main parts and with respect to the 
123 124 125 126 137 following:

correct order as well:

1. the objective function to be optimized (either maximized or minimized);

2. the (sets of) constraints, or else the relationships between the decision variables and coefficients, right-hand side (RHS);

3. the decision variables and their domain space.

We used the programming environment of Python coupled with its modeling library, namely Pyomo. Similar approaches in terms of software selection have been presented for Differential and Algebraic Equations (DAE) modeling and optimization in (Nicholson et al., 2018; Nikolić, 2016). By combining Python and Pyomo we have the ability to transform a simplified representation of a mathematical model initially written in $\mathrm{AT}_{\mathrm{E} X}$ into a formal AML formulation and eventually optimize it. In other words, the platform reads $\mathrm{LT}_{\mathrm{E}} \mathrm{X}$ code and then writes Pyomo abstract models or the code generates code. The resulting .py file is usable outside of the platform's frame, thus not making the binding and usage of these two necessary after conversion. The main components that we employed for this purpose are the

- Front-end: HTML, JavaScript, MathJax ${ }^{4}$ and Google Polymer ${ }^{5}$;

- Back-end: Python with Django ${ }^{6}$ and Pyomo.

In order to increase the effectiveness and user-friendliness of the platform, a Graphical-User Interface (GUI) based on HTML, JavaScript (front-end) and Django as the web-framework (back-end) has been implemented, as shown in Figure 2. The user-input is facilitated mainly via Polymer objects ${ }^{7}$. As the main feature of the platform is to allow modeling in $\mathrm{AT}_{\mathrm{E} X} \mathrm{X}$ language, we used MathJax as the rendering engine. In this way, the user can see the compiled version of the input model. All of these components form a single suite that works across different computational environments. The front-end is plain but incorporates

\footnotetext{
${ }^{4}$ https://www.mathjax.org/

${ }^{5}$ https://www.polymer-project.org/

${ }^{6}$ https://www.djangoproject.com/

${ }^{7}$ https://www.polymer-project.org/
} 
147 the necessary functionality for input and output, as well as some solver options. The role

148 of the back-end is to establish the communication between the GUI and the parser with the

149 functions therein. In this way the inputs are being processed inside Python in the back-

150 ground, and the user simply witnesses a seamless working environment without having to

151 understand the black-box parser in detail.

152 The main components of the GUI are:

153 - Abstract model input: The input of the $\mathrm{AT}_{\mathrm{E} X} \mathrm{X}$ model, either directly inside the Polymer

154 input text-box or via file upload (a tex containing the required source $\mathrm{ATEX}_{\mathrm{E}}$ code)

155 - Data files: The input of the data set which follows the abstract definition of the model

156 via uploading the AMPL-format (.dat) data file

- Solver options: An array of solver - related options such as:

158

1. NEOS server job using CPLEX

159

2. Solve the relaxed LP (if MILP)

160

3. Select GPLK (built-in) as the optimization solver

161

4. Select CPLEX (if available) as the optimization solver (currently set to default)

162

The following is an example of a ${ }^{A} T_{\mathrm{E}} \mathrm{X}$ formulated optimization problem which is ready

163 to use with the platform; the well-known Traveling Salesman Problem (TSP) (Applegate 164 et al., 2007):

$$
\begin{aligned}
& \text { minimize } \quad \sum_{i, j: i \neq j}\left(d_{i, j} x_{i, j}\right) \\
& \text { subject to: } \\
& \sum_{j: i \neq j}\left(x_{i, j}\right)=1 \quad \forall i \\
& \sum_{i: i \neq j}\left(x_{i, j}\right)=1 \quad \forall j \\
& u_{i}-u_{j}+n x_{i, j} \leq n-1 \quad \forall i \geq 2, j \leq|j|-1, i \neq j \\
& u \in Z, x \in\{0,1\}
\end{aligned}
$$

165 and the raw $\mathrm{LT}_{\mathrm{E}} \mathrm{X}$ code used to generate this was: 


\section{=IMLI}

A Mathematical Programming Modelling Platform

Enter your optimization problem in $L F T_{E} X:$ Example

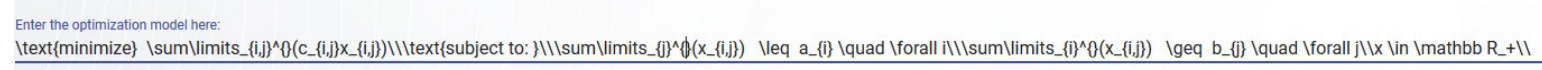

$\operatorname{minimize} \sum_{i, j}\left(c_{i, j} x_{i, j}\right)$

subject to:

$\sum_{j}\left(x_{i, j}\right) \leq a_{i} \quad \forall i$

$\sum_{i}\left(x_{i, j}\right) \geq b_{j} \quad \forall j$

$x \in \mathbb{R}_{+}$

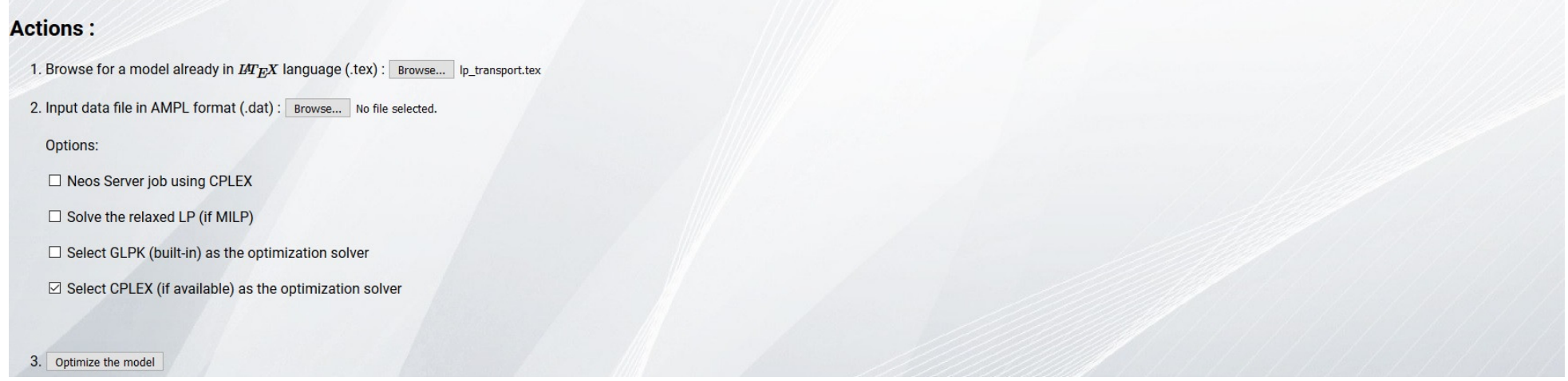

Figure 2: The simplified Graphical User Interface (GUI). The GUI contains the basic but fundamental options to use the platform, such as model input, solver selection and solution extraction. 


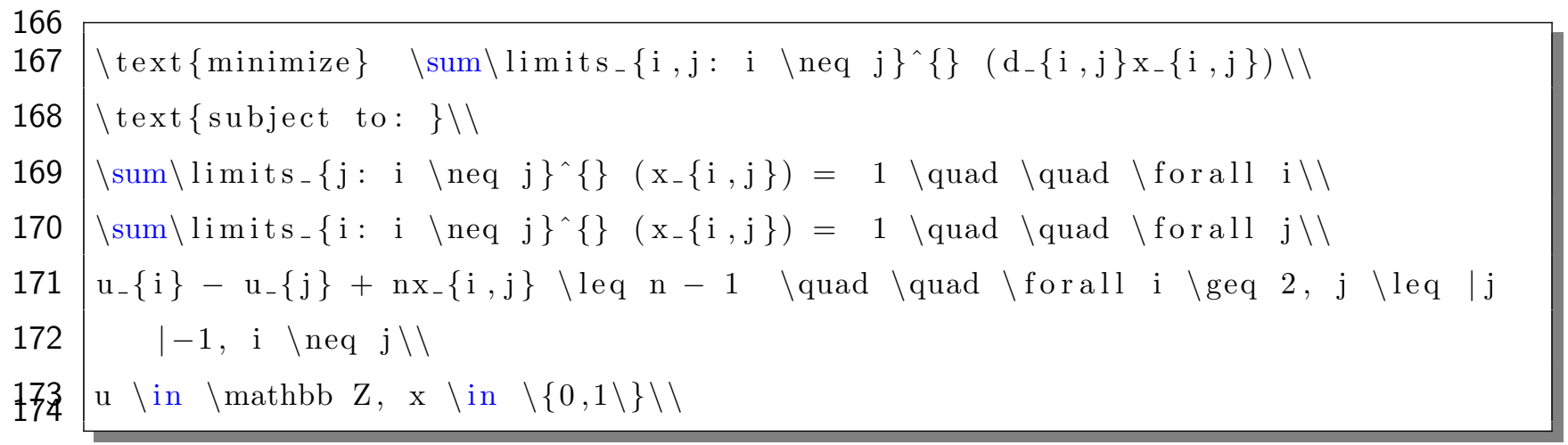

175 which is the input for the platform. The user can either input this code directly inside the

176 Google polymer text box or via a pre-made .tex file which can be uploaded in the corre-

177 sponding field of the GUI. Either way, the MathJax Engine then renders $\mathrm{AT}_{\mathrm{E}} \mathrm{X}$ appropriately

178 so the user can see the resulting compiled model live. Subject to syntax-errors, the MathJax

179 engine might or might not render the model eventually, as naturally expected. Empty lines

180 or spaces do not play a role, as well as commented-out lines using the standard notation (the

181 percentage symbol \%). The model file always begins with the objective function sense, the

182 function itself, and then the sets of constraints follow, with the variables and their respective

183 type at the end of the file.

\section{3. Parser - Execution Engine}

185 As parser we define the part of the code (a collection of Python functions) in the back-end 186 side of the platform which is responsible for translating the model written in $\mathrm{ET}_{\mathrm{EX}}$ to Py187 omo, the modeling component of the Python programming language. In order to effectively 188 translate the user model input from $\mathrm{AT}_{\mathrm{EX}}$, we need an array of programming functions to 189 carry out the conversion consistently since preserving the equivalence of the two is implied.

190 The aim of the implementation is to provide minimum loss of generality in the ability to 191 express mathematical notation for different modeling needs.

192 A detailed description of the implemented scheme is given in Figure 3. A modular design 193 of different functions implemented in Python and the established communication of those 194 (exchanging input and output-processed data) form the basic implementation concept. This 195 type of design allows the developers to add functionality in a more clear and effective way.

196 For instance, to upgrade the parser and support Mixed Integer Quadratic Programming 
197 (MIQP) problems, an update only to the parsing function assigned to convert the optimiza198 tion objective function is required.

199 Once the .tex model file and the .dat AMPL formatted data file are given, the platform 200 then starts processing the model. The conversion starts by reading the variables of the model 201 and their respective types, and then follows with component identification (locating the 202 occurrence of the variables in each constraint) and their inter-relationships (multiplication, 203 division, summation etc.). Additionally, any summation and constraint conditional indexing 204 schemes will be processed separately. Constraint-by-constraint the parser gradually builds 205 the .py Pyomo abstract model file. It then merges through Pyomo the model with its data 206 set and calls the selected solver for optimization.

\subsection{Pre-processing}

A significant amount of pre-processing takes place prior of parsing. The minimum and essential is to first tidy up the input; that is, clear empty lines and spaces, as well as reserved (by the platform) keywords that the user can include but do not play any role in functional parsing (such as the \quad command). The platform also supports the use of Greek letters. For instance if a parameter is declared as $\alpha$ the platform identifies the symbol, removes the backslash and expects to find alpha in the data-file. This takes place also in the preprocessing stage.

The user can also opt-out selectively the constraints by putting regular comments in

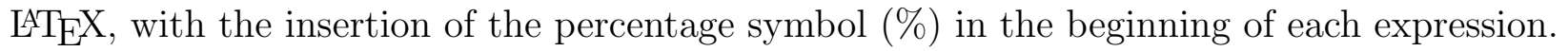
Once done, we attempt to simplify some types of mathematical expressions in order to be able to better process them later on. More specifically, we have two main functions that handle fractions and common factor (distributive expressions) simplifications. For example:

$$
\frac{A_{i} B_{j}}{D_{i}} \text { is then converted to: }\left(A_{i} B_{j}\right) / D_{i}
$$

and

$$
\beta(\alpha+1) \text { is converted as expected to: } \beta \alpha+\beta
$$

When handling fractions, the user can employ the frac environment to generate them; the parser in the background always though processes the analytic form (the same applies with 


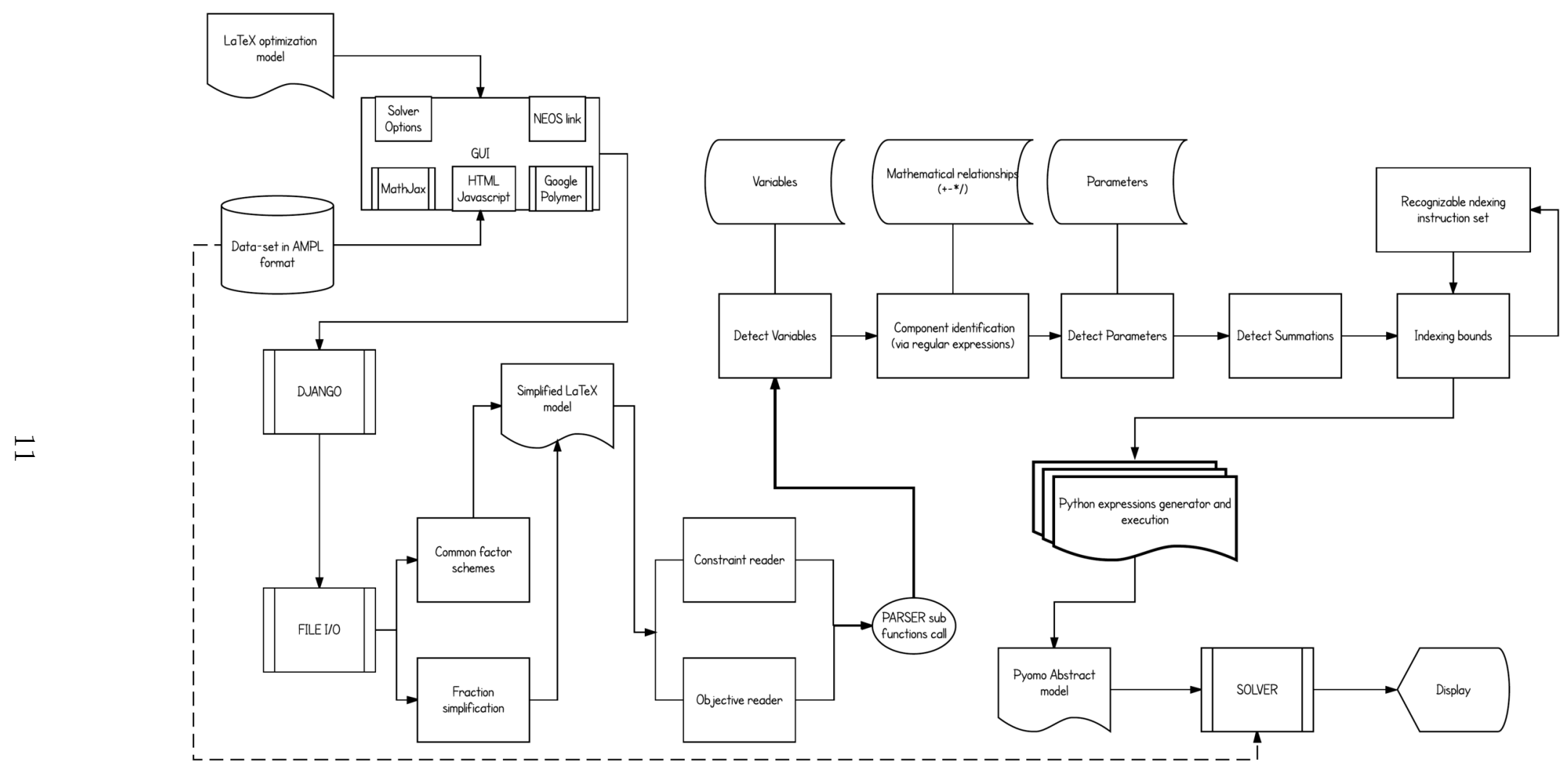

Figure 3: The overall flow of the implementation. From user input to solving the optimization problem or simply exporting the equivalent Pyomo model file. 
225 the distributive form of multiplications), no matter if the initial input was done using the 226 frac environment.

227 This keeps the basic component identification functions intact, since their input is trans-

228 formed first to the acceptable analytical format. Instead of transforming the parsing func229 tions, we transform the input in the acceptable format. However, the user does not lose 230 neither functionality nor flexibility, as this takes place in the background. To put it simply, 231 either the user inputs the analytic form of an expression or the compact, the parser is still 232 able to function correctly.

233 To frame the capabilities of the parser, we will now describe how the user can define 234 optimization models in the platform with a given example and the successful parsing to 235 Pyomo. The parser first attempts to split the model into its three major distinct parts:

- the objective function

- the sets of constraints These three parts are in a way independent but interconnected as well.

\subsection{Processing Variables}

241 The parser first attempts to read the variables and their respective domain space (type).

242 The platform is case sensitive since it is based on Pyomo. The processing is done using string 243 manipulation functions, therefore the use of regular expressions in Python was essential and 244 effective.

245 Reasonably, the focus was on consistency and reliability, rather computational perfor246 mance mainly due to the lightweight workload of the processing demands in general. In 247 order to do that, the parser uses keywords as identifiers while scanning from the top to the 248 bottom of the manually curated tex file which contains the abstract model in ATEX. For 249 the three respective different parts mentioned earlier, the corresponding identifiers are:

250 1. Objective function: $\{$ minimize, maximize

251 2. Sets of constraints: $\{\backslash l e q, \backslash g e q,=\}$ 
253 This helps separate the processing into sections. Each section is analyzed and passes the 254 information in Pyomo syntax in the .py output model file. Variable types can appear in the 255 following way:

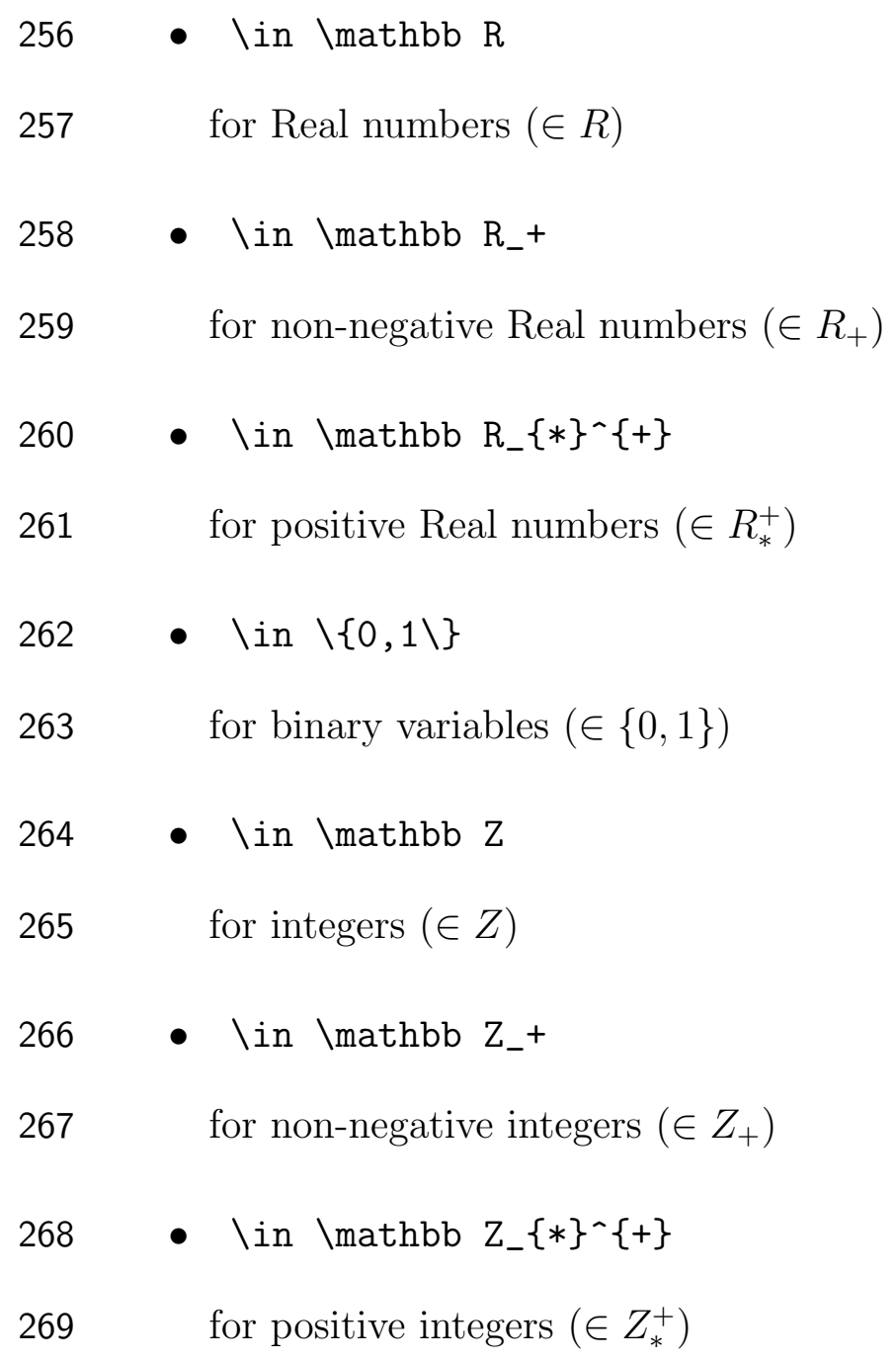

270 In order to avoid confusion between lowercase and uppercase, the identifiers are converted 271 to uppercase prior of comparison. Upon locating these keywords, the parser separates the 272 processing and starts calling the corresponding functions. Once the variables and their 273 types are processed (expected to be found at the bottom of the mathematical definition of 274 the model), the parser then creates a list of strings for the names of the variables. This 275 is one of the crucial structures of the parser and utilized alongside the entire run-time of 
276 the conversion process. A list of the same length, which holds the types of each respective

277 variable, is also created. The platform in general uses Python lists to store information about

278 variables, index sets, parameters, scalars etc.

279

280

281

282

283

284

285

286 287

288

289

290

291

292

293

294

295

296

297

298

299

300

301

302

\subsection{Decomposing constraints and objective function expressions}

Our approach for understanding the inter-mathematical relationships between the variables and the parameters relied on exploiting the fundamental characteristics of Linear Programming:

\section{- Proportionality}

- Additivity

- Divisibility

These mathematical relationships can help us understand the structure of the expressions and how to decompose them. By decomposition we define the fragmentation of each mathematical expression at each line of the .tex input model file into the corresponding variables, parameters, summations etc. so as we can process the given information accordingly. A simple graphical example is given in Figure 4.

The decomposition with the regular expressions is naturally done via the strings of the possible operators found, that is: addition, subtraction, division $(+,-, /)$, since the asterisk to denote multiplication $(*$ or $\cdot$ ) is usually omitted in the way we describe the mathematical expressions (e.g. we write $a x$ to describe coefficient $a$ being multiplied by variable $x$ ). In some cases however it is imperative to use the asterisk to decompose a multiplication. For example, say $D s$ is a parameter and $s$ is also a variable in the same model. There is no possible way to tell whether the expression $D s$ actually means $D^{*} s$ or if it is about a new parameter altogether, since the parameters are not explicitly defined in the model definition (as in AMLs). Adding to that the fact that for the scalars there is no associated underscore character to identify the parameter as those are not associated with index sets, the task is even more challenging. Therefore, we should write $D^{*} s$ if $D$ is a scalar. As for parameters with index sets, for example $D s_{i} s_{i}$ causes no confusion for the parser because the 


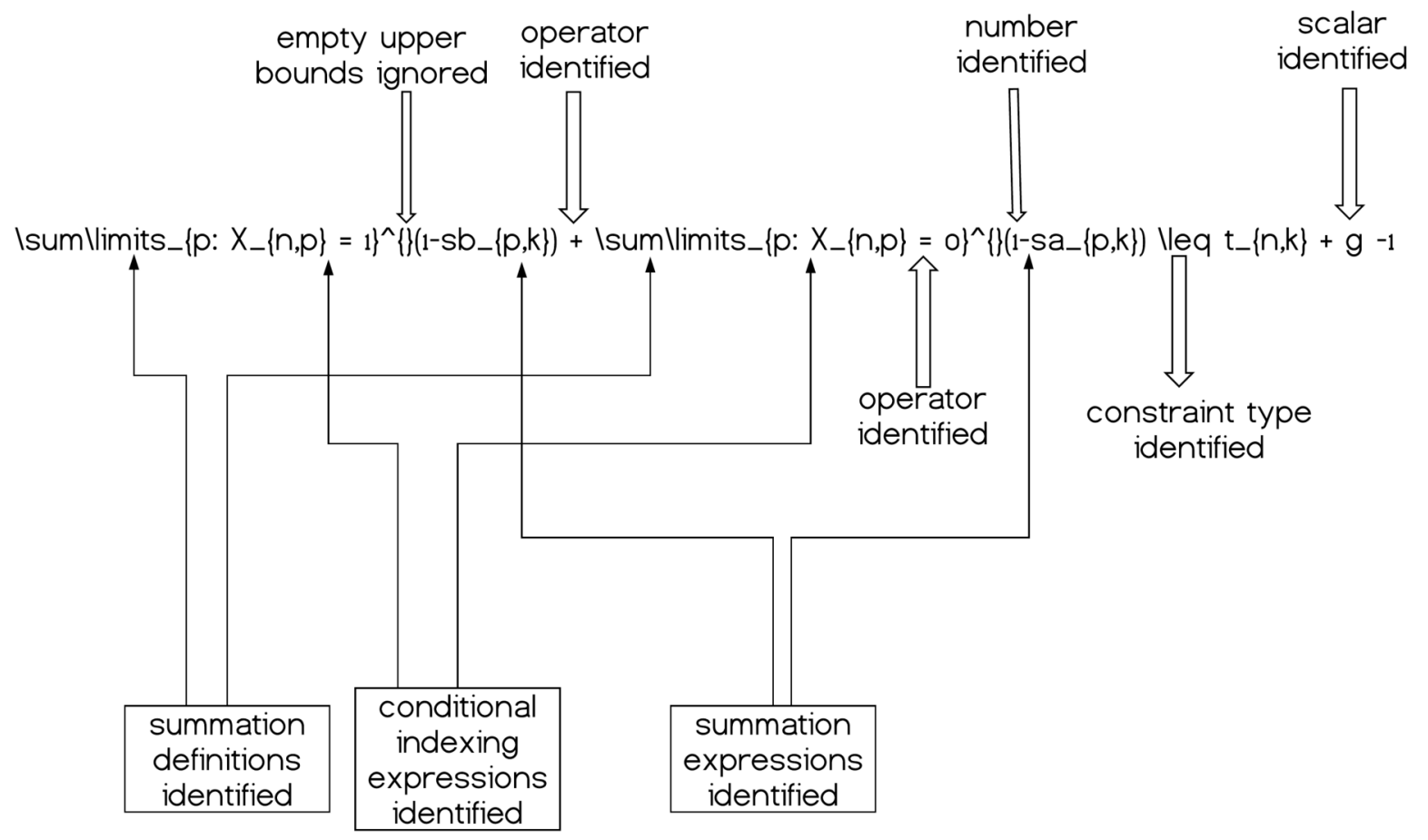

Figure 4: A simple constraint having its components (partially) decomposed and therefore identified; summations, operators, scalars and numerical quantities.

303 decomposition based on the underscore character clearly reveals two separate components.

304 In this way, the platform also identifies new parameters. This means that since we know 305 for instance that $s$ is a variable but $D s$ is not we can dynamically identify $D s$ on the fly 306 (as we scan the current constraint) as being a parameter which is evidently multiplied with 307 variable $s$, both having index set $i$ associated with them. However, we need to pay attention 308 on components appearing iteratively in different or in the same sets of constraints; did we 309 have the component already appearing previously in the model again? In that case we do 310 not have to declare it again in the Pyomo model as a new quantity, as that would cause a 311 modeling error.

312 By declaration we mean the real-time execution of a Python command that creates the 313 associated terms inside the Pyomo abstract objected-oriented (OO) model. For instance if 314 a set $i$ is identified, the string model. $i=\operatorname{Set}(\operatorname{dimen}=1)$ is first written inside the text 315 version of the Pyomo model file, and then on-the-fly executed independently inside the 316 already parsing Python function using the exec command. The execution commands run in 
317 a sequential manner. All the different possible cases of relationships between parameters and 318 variables are dynamically identified, and the parser keeps track of the local (per constraint) 319 and global (per model) list of parameters identified while scanning the model in dynamically 320 growing lists.

321 Dynamic identification of the parameters and index sets is one of the elegant features of 322 the platform, since in most Algebraic Modeling Languages (AMLs) the user explicitly defines 323 the model parameters one-by-one. In our case, this is done in an intelligent automated 324 manner. Another important aspect of the decomposition process is the identification of the 325 constraint type $(<=,=,>=)$, since the position of the operator is crucial to separate the 326 left and the right hand side of the constraint. This is handled by an independent function. 327 Decomposition also helps identify Quadratic terms. By automatic conversion of the caret 328 symbol to $* *$ (as this is one of the ways to denote power of a variable in Pyomo language)

329 the split function carefully transfers this information intact to the Pyomo model.

\subsection{Summations and conditional indexing}

Summation terms need to be enclosed inside parentheses $(\cdots)$, even with a single component. This accelerates identification of the summation terms with clarity and consistency. Summations are in a way very different than processing a simplified mathematical expression in the sense that we impose restrictions on how a summation can be used. First of all, the corresponding function to process summations tries to identify how many summation expressions exist in each constraint at a time. Their respective indexing expressions are extracted and then sent back to the index identification functions to be processed. The assignment of conditional indexing with the corresponding summation is carefully managed. Then, the summation commands for the Pyomo model file are gradually built. Summations can be expressed in the following form, and two different fields can be utilized to exploit conditional indexing (upper and lower brackets):

$\backslash \operatorname{sum} \backslash \operatorname{limits} s_{-}\left\{\mathrm{p}: \mathrm{X}_{-}\{\mathrm{n}, \mathrm{p}\}=1\right\}^{\wedge}\{\}\left(1-\mathrm{sb}_{-}\{\mathrm{p}, \mathrm{k}\}\right)$

which then compiles to: $\sum_{p: X_{n, p}=1}\left(1-s b_{p, k}\right)$

This means that the summation will be executed for all values of $p$, (that is for $p=1:|p|$ ) 
347 but only when $X_{n, p}=1$ at the same time. If we want to use multiple and stacked summations

348 (double, triple etc.) we can express them in the same way by adding the indexes for which

349 the summation will be generated, as for example:

350

352

$\backslash \operatorname{sum} \backslash \operatorname{limits}_{-}\{\mathrm{i}, \mathrm{j}\}^{\wedge}\{\}\left(\mathrm{X}_{-}\{\mathrm{i}, \mathrm{j}\}\right)$

353 which then compiles to: $\sum_{i, j}\left(X_{i, j}\right)$

354

355 and will run for the full cardinality of sets $i, j$. Dynamic (sparse) sets imposed on constraints

356 can be expressed as:

357

$35 \%$

$X_{-}\{i, j\}=Y_{-}\{i, j\} \backslash$ forall $(i, j) \backslash$ in $C \backslash \backslash$

360 which then compiles to: $X_{i, j}=Y_{i, j} \quad \forall(i, j) \in C$

361

362 This means that the constraint is being generated only for those values of $(i, j)$ which belong

363 to the dynamic set $C$. In order to achieve proper and precise processing of summations

364 and conditional indexing, we have built two separate functions assigned for the respective

365 tasks. Since specific conditional indexing schemes can take place both for the generation

366 of an entire constraint or just simply for a summation inside a constraint, two different

367 sub-functions process this portion of information. This is done using the $\backslash$ forall command

368 at the end of each constraint, which changes how the indexes are being generated for the

369 vertical expansion of the constraints from a specific index set. Concerning summations it is

370 done with the bottom bracket information for horizontal expansion, as we previously saw

371 for instance with $p: X_{n, p}=1$.

372 A series of challenges arise when processing summations. For instance, which components

373 are inside a summation symbol? A variable that might appear in two different summations

374 at the same constraint can cause confusion. Thus, using a binary list for the full length

375 of variables and parameters present in a constraint we identify the terms which belong to

376 each specific summation. This binary list gets re-initialized for each different summation

377 expression. From the lower bracket of each summation symbol, the parser is expecting to

378 understand the indexes for which the summation is being generated. This is done by either 
379 simply stating the indexes in a plain way (for instance $a, b$ or if a more complex expression 380 is used, the for-loop indexes for the summations are found before the colon symbol (:).

\section{3.5. Constraint indexing}

382 At the end of each constraint, the parser identifies the " $\forall$ "' ( $\backslash$ forall) symbol which then 383 helps understand for which indexes the constraints are being sequentially generated (vertical 384 expansion). For instance $\forall(i, j) \in C$ makes sure that the constraint is not generated for all 385 combinations of index sets $i, j$, but only the ones appearing in the sparse set $C$. The sparse 386 sets are being registered also on the fly, if found either inside summation indexing brackets 387 or in the constraint general indexing (after the $\forall$ symbol) by using the keywords $\backslash$ in, $\backslash$ notin. 388 The simplest form of constraint indexing is for instance:

$$
\sum_{j: i \neq j}\left(x_{i, j}\right)=1 \quad \forall i
$$

390 391 392 393 394 395 396 397 398

where the constraint is vertically expanding for all elements of set $i$ and the summation is running for all those values of set $j$ such that $i$ is not equal to $j$. More advanced cases of constraint conditional indexing are also identified, as long as each expression is separated with the previous one by using a comma. For example in:

$$
\forall i<|i|, j \geq i+1
$$

we see each different expression separated so the parser can process the corresponding indexing. Three different functions handle identification on constraint- level and the input for the general function that combines these three, accepts as input the whole expression. We process each component (split by commas) iteratively by these three functions:

1. to identify left part (before the operator/reserved keyword/command)

2. the operator and

3. the right-hand part

2 For example, in $i<|i|$, the left part is set $i$, the operator is $<$ and the right-hand part is the cardinality of set $i$. In this way, by adding a new operator in the acceptable operators list inside the code, we allow expansion of supported expressions in a straightforward manner. 


\section{4. An illustrative parsing example}

406 Let us now follow the sequential steps that the parser takes to convert a simple example. 407 Consider the well-known transportation problem:

$$
\begin{array}{ll}
\operatorname{minimize} & \sum_{i, j}\left(c_{i, j} x_{i, j}\right) \\
\text { subject to: } & \\
& \sum_{j}\left(x_{i, j}\right) \leq a_{i} \quad \forall i \\
& \sum_{i}\left(x_{i, j}\right) \geq b_{j} \quad \forall j \\
& x \in R_{+}
\end{array}
$$

408 We will now provide in-depth analysis of how each of the main three parts in the model can 409 be processed.

410

411

412

413

414

415

416 420 found and their respective types.

\subsection{Variables}

The parser first attempts to locate the line of the tex model file that contains the variable symbols and their respective domains. This is done by trying to identify any of the previously presented reserved keywords specifically for this section. The parser reaches the bottom line by identifying the keyword mathbbR + in this case. Commas can separate variables belonging to the same domain, and the corresponding parsing function splits the collections of variables of the same domain and processes them separately.

In this case, the parser identifies the domain and then rewinds back inside the string expression to find the variable symbols. It finds no commas, thus we collect only one variable with the symbol $x$. The platform then builds two Python lists with the name of the variables

421

422

423

424 425 426

\subsection{Objective function}

The parser then reads the optimization sense (by locating the objective function expression using the keywords, in this case minimize) and tries to identify any involved variables in the objective function. In a different scenario, where not all of the model variables are present in the objective function, a routine identifies one-by-one all the remaining variables and their associated index sets in the block of the given constraint sets. 
427

428

429

430

431

432

433

434

The parser first attempts to locate any summation symbols. Since this is successful, the contained expression is extracted as $c_{\{i, j\}} x_{\{i, j\}}$, by locating the parentheses bounds () . In case of multiple summations, or multiple expressions inside the parentheses, we process them separately. The bounds of the summation symbol (the lower and upper brackets) respectively will be analyzed separately. In this case, the upper one is empty, so the lower one contains all the indexes for which the summation has to scale. Separated by commas, a simple extraction gives $i, j$ to be used for the Pyomo for-loop in the expression. There is no colon identified inside the lower bracket of the summation, thus no further identification of conditional indexing is required.

A split function is then applied on the extracted mathematical expression $c_{-}\{i, j\} x_{-}\{i, j\}$ to begin identification of the involved terms. Since there are no operators $(*,+,-, /)$ we have a list containing only one item; the combined expression. It follows that the underscore characters are used to frame the names of the respective components. It is easy to split on these characters and then create a list to store the pairs of the indexes for each component. Thus, a sub-routine detects the case of having more than just one term in the summationextracted expression. In this example, $c$ is automatically identified as a parameter because of its associated index set which was identified with the underscore character and since it does not belong to the list of variables.

The global list of parameters is then updated by adding $c$, as well as the parameters for the current constraint/objective expression. This helps us clarify which parameters are present in each constraint as well as the set of parameters (unique) for the model thus far, as scanning goes on. Once the parameter $c$ and variable $x$ are identified and registered with their respective index sets, we proceed to read the constraint sets. The parser creates expressions as the ones shown below for this kind of operations:

model. $\mathrm{i}=\operatorname{Set}(\operatorname{dimen}=1) \backslash \backslash$ model. $\mathrm{j}=\operatorname{Set}(\operatorname{dimen}=1) \backslash \backslash$ model. $\mathrm{c}=$ Param $(\operatorname{model} . \mathrm{i}, \operatorname{model} . \mathrm{j}$, initialize $=0) \backslash \backslash$ model. $\mathrm{x}=\operatorname{Var}(\operatorname{model} . \mathrm{i}, \operatorname{model} . \mathrm{j}$, domain=NonNegativeReals $) \backslash \backslash$

Since the objective function summation symbol was correctly identified with the respective indexes, the following code is generated and executed: 
459

460

461

462

463

465

def obj_expression (model):

$\operatorname{model} . F=\operatorname{sum}(\operatorname{model} . c[i, j] * \operatorname{model} . x[i, j]$ for $i$ in model. $i$ for $j$ in model.j $)$ return model.F

model.OBJ $=$ Objective $($ rule=obj_expression,, sense $=$ minimize $)$

466

467

468

469

470

471

472

473

474

475

476

476

479

480

481

482

483

484

485

487

488

489

490

491

492

493

\subsection{Constraints}

Since the constraints sets are very similar, for shortness we will only analyze the first one. The parser first locates the constraint type by finding either of the following operators $\leq, \geq,=$. It then splits the constraint in two parts, left and right across this operator. This is done to carefully identify the position of the constraint type operator for placement into the Pyomo constraint expression later on.

The first component the parser gives is the terms identified raw in the expression $\left(\left[{ }^{\prime} x_{i, j}^{\prime},{ }^{\prime} a_{i}^{\prime}\right]\right)$. Parameter $a$ is identified on the fly and since $x$ is already registered as a variable and the parser proceeds to only register the new parameter by generating the following Pyomo expressions:

model.a $=$ Param (model.i, initialize $=0$ )

def axb_constraint_rule_1 (model, X):

and the full resulting function is:

def axb_constraint_rule_1 (model, i ) : $\operatorname{model.C} 1=\operatorname{sum}(\operatorname{model} \cdot \mathrm{x}[\mathrm{i}, \mathrm{j}]$ for $\mathrm{j}$ in $\operatorname{model} . \mathrm{j})<=\operatorname{model} . \mathrm{a}[\mathrm{i}]$ return model. C_1 model. AxbConstraint_1=Constraint (model.i, rule=axb_constraint_rule_1) 
495 5. Discussion

496 Developing a parser that would be able to understand almost every different way of 497 writing mathematical models using $\mathrm{AT}_{\mathrm{E} X}$ is nearly impossible; however, even by framing 498 the way the user could write down the models, there are some challenges to overcome. 499 For instance, the naming policy for the variables and parameters. One would assume that 500 these would cause no problems but usually this happens because even in formal modeling 501 languages, the user states the names and the types of every component of the problem. 502 Starting from the sense of the objective function, to the names and the types of the variables 503 and parameters as well as their respective sizes and the names of the index sets, everything 504 is explicitly defined. This is not the case though in this platform; the parser recognizes the 505 parameters and index sets with no prior given information. This in turn imposes trade-offs 506 in the way we write the mathematical notation. For instance multiple index sets have to be 507 separated by commas as in $x_{i, j}$ instead of writing $x_{i j}$.

508 On the other hand, using symbolic representation of the models in $\mathrm{AT}_{\mathrm{EX}}$ can enable the 509 user quickly identify errors in the description of the model, the involved variables, parameters 510 or their mathematical relationships therein. This as opposed trying to debug models that 511 have been developed directly in a programming language or in an AML, which would make 512 the detection of such errors or typos more challenging.

513 By scanning a constraint, the parser quickly identifies as mentioned the associated vari514 ables. In many cases parameters and variables might have multiple occurrences in the same 515 constraint. This creates a challenging environment to locate the relationships of the param516 eters and the variables since they appear in multiple locations inside the string expressions 517 and in different ways. On top of this, the name of a parameter can cause identification prob518 lems because it might be a sub/super set of the name of another parameter, e.g. parameter $519 \mathrm{AB}$, and parameter ABC. Therefore naming conflicts are carefully resolved by the platform 520 by meticulously identifying the exact location and occurrences of each term.

521 The cpu time required for each step in the modeling process of the platform (conversion 522 from $\mathrm{AT}_{\mathrm{E} X} \mathrm{X}$ to Pyomo, Pyomo model generation, Solver) can be found in the Supplementary 523 Information. It can be noted that the parser is the least consuming step, which clearly demon524 strates the efficiency of the platform. The Pyomo model generation and solver (CPLEX in 
525 526 527 528

530

531 532

our measurements) steps and their associated cpu-time are completely outside of the parser's control. However, it is essential to get an idea of how these timings compare to each other with the addition of this extra higher level of abstraction in the beginning of the modeling process.

Challenges also arise in locating which of the terms appearing in a constraint belong to summations, and to which summations; especially when items have multiple occurrences inside a constraint, there needs to be a unique identification so as to include a parameter (or a variable) inside a specific summation or not. We addressed this with the previously introduced binary lists. Then for each of those summation symbols, the items activated (1) are included in the summation or not (0) and the list is generated for each different summation within the expression.

Additionally, another challenge constitutes the extension of the platform to support nonlinear terms, where each term itself can be a combination of various operators and mathematical functions.

Finally, it is worth mentioning that the amount of lines/characters to represent a model in $\mathrm{AT}_{\mathrm{E} X i n}$ comparison with the equivalent model in Pyomo is substantially smaller. In this respect, the platform accelerates the modeling development process.

\section{Conclusions}

We presented a platform for rapid model generation using $\mathrm{AT}_{\mathrm{E}} \mathrm{X}$ as the input language for mathematical programming, starting with the classes of LP, MILP and MIQP. The platform is based on Python and parses the input to Pyomo to successfully solve the underlying optimization problems. It uses a simple GUI to facilitate model and data input based on Django as the web-framework. The user can exploit locally installed solvers or redirect to NEOS server. This prototype platform delivers transparency and clarity, speedup of the

549 model design and development process (by significantly reducing the required characters to 50 type the input models) and abstracts the syntax from programming languages and AMLs.

51 It therefore delivers reproducibility and the ability to replicate scientific work in an effective 52 manner from an audience not necessarily versed in coding. Future work could possibly involve expansion to support nonlinear terms as well as differential and algebraic equations, sanity 
554 checking and error catching on input, the ability to embed explanatory comments in the input

555 model file which would transfer to the target AML, extending the functionality concerning

556 bounds on the variables as well as adding further support to built-in $\mathrm{AT}_{\mathrm{E}} \mathrm{X}$ commands (such 557 as $\backslash$ left $[$ ) which would capture more complex mathematical relationships.

\section{7. Acknowledgments}

559 We would like to thank Prof. Eric Fraga and Dr. Aristotelis Kittas for useful discussions.

560

561

562

563

564

565

566

567

568

569

570

571

572

573

574

575

576

577

578

579

580

581

582

583

584

585

\section{References}

Applegate, D.L., Bixby, R.E., Chvatal, V., Cook, W.J., 2007. The Traveling Salesman Problem: A Computational Study (Princeton Series in Applied Mathematics). Princeton University Press, Princeton, NJ, USA.

Bertsimas, D., Tsitsiklis, J., 1997. Introduction to Linear Optimization. Third printing edition ed., Athena Scientific.

Bisschop, J., Roelofs, M., 2011. AIMMS language reference, version 3.12. Paragon Decision Technology .

Bruce A. McCarl et. al., 2013. McCarl Expanded GAMS User Guide, GAMS Release 24.2.1. GAMS Development Corporation. Washington, DC, USA. URL: http://www.gams.com/mccarl/mccarlhtml/ gams_user_guide_2005.htm.

Cohen, M.C., Leung, N.H.Z., Panchamgam, K., Perakis, G., Smith, A., 2017. The impact of linear optimization on promotion planning. Operations Research 65, 446-468. URL: https://doi.org/10.1287/opre . 2016.1573, doi:10.1287/opre.2016.1573, arXiv:https://doi.org/10.1287/opre.2016.1573.

Dunning, I., Huchette, J., Lubin, M., 2017. Jump: A modeling language for mathematical optimization. SIAM Review 59, 295-320. URL: https://doi.org/10.1137/15M1020575, doi:10.1137/15M1020575, arXiv:https://doi.org/10.1137/15M1020575.

Editorial, 2014. Software with impact. Nature Methods 11. URL: http://dx.doi.org/10.1038/nmeth. 2880, doi:10.1038/nmeth. 2880 .

Erik, E., Christian, H., Markus, I., David, M., Sandra, F., Gregor, T., Henning, B., Günter, W., Jens-Uwe, R., . MOSAIC - enabling large-scale equation-based flow sheet optimization. Chemie Ingenieur Technik 89, 620-635. URL: https://onlinelibrary.wiley.com/doi/abs/10.1002/cite.201600114, doi:10.1002/ cite.201600114, arXiv:https://onlinelibrary.wiley.com/doi/pdf/10.1002/cite.201600114.

Fourer, R., Gay, D., Kernighan, B., 1993. AMPL: A Modeling Language for Mathematical Programming. Scientific Press. URL: https://books .google.co.uk/books?id=8vJQAAAMAAJ.

Hart, W.E., Laird, C.D., Watson, J.P., Woodruff, D.L., Hackebeil, G.A., Nicholson, B.L., Siirola, J.D., 2017. Pyomo-optimization modeling in python. volume 67. Second ed., Springer Science \& Business Media. 
586 Hart, W.E., Watson, J.P., Woodruff, D.L., 2011. Pyomo: modeling and solving mathematical pro-

587

588

589

590

591

592

593

594

595

596

597

598

599

600

601

602

603

604

605

606

607

608

609

610

611

612

613

614

615

616

617

618

619

620

621 grams in python. Mathematical Programming Computation 3, 219. URL: https://doi.org/10.1007/ s12532-011-0026-8, doi:10.1007/s12532-011-0026-8.

Jackson, M., 2012. Aspects of abstraction in software development. Software \& Systems Modeling 11, 495-511. URL: https://doi.org/10.1007/s10270-012-0259-7, doi:10.1007/s10270-012-0259-7.

Kallrath, J., 2004. Modeling Languages in Mathematical Optimization (APPLIED OPTIMIZATION). Kluwer Academic Publishers, Norwell, MA, USA.

Knuth, D.E., 1984. Literate programming. Comput. J. 27, 97-111. URL: http://dx.doi.org/10.1093/ comjnl/27.2.97, doi:10.1093/comjnl/27.2.97.

Kratica, J., Dugošija, D., Savić, A., 2014. A new mixed integer linear programming model for the multi level uncapacitated facility location problem. Applied Mathematical Modelling 38, 2118 - 2129. URL: http:// www.sciencedirect.com/science/article/pii/S0307904X13006240, doi:https://doi.org/10.1016/ j.apm.2013.10.012.

Leek, J.T., Peng, R.D., 2015. Opinion: Reproducible research can still be wrong: Adopting a prevention approach. Proceedings of the National Academy of Sciences 112, 16451646. URL: http://www.pnas.org/content/112/6/1645, doi:10.1073/pnas.1421412111, arXiv:http://www.pnas.org/content/112/6/1645.full.pdf.

Liu, S., Papageorgiou, L.G., 2018. Fair profit distribution in multi-echelon supply chains via transfer prices. Omega 80, 77 - 94. URL: http://www.sciencedirect.com/science/article/pii/ S0305048316307897, doi:https://doi.org/10.1016/j.omega.2017.08.010.

Lubin, M., Dunning, I., 2015. Computing in operations research using Julia. INFORMS Journal on Computing 27, 238-248. URL: https://doi.org/10.1287/ijoc.2014.0623, doi:10.1287/ijoc.2014.0623, arXiv:https://doi.org/10.1287/ijoc.2014.0623.

Melas, I.N., Samaga, R., Alexopoulos, L.G., Klamt, S., 2013. Detecting and removing inconsistencies between experimental data and signaling network topologies using integer linear programming on interaction graphs. PLOS Computational Biology 9, 1-19. URL: https://doi.org/10.1371/journal.pcbi. 1003204, doi:10.1371/journal.pcbi.1003204.

Mitsos, A., Melas, I.N., Siminelakis, P., Chairakaki, A.D., Saez-Rodriguez, J., Alexopoulos, L.G., 2009. Identifying drug effects via pathway alterations using an integer linear programming optimization formulation on phosphoproteomic data. PLOS Computational Biology 5, 1-11. URL: https://doi.org/10.1371/ journal.pcbi.1000591, doi:10.1371/journal.pcbi.1000591.

Mouha, N., Wang, Q., Gu, D., Preneel, B., 2012. Differential and Linear Cryptanalysis Using MixedInteger Linear Programming. Springer Berlin Heidelberg, Berlin, Heidelberg. pp. 57-76. URL: https: //doi.org/10.1007/978-3-642-34704-7_5, doi:10.1007/978-3-642-34704-7_5.

Nicholson, B., Siirola, J.D., Watson, J.P., Zavala, V.M., Biegler, L.T., 2018. pyomo.dae: a modeling and automatic discretization framework for optimization with differential and algebraic equations. Mathemat- 
622

623

624

625

626

627

628

629

630

631

632

633

634

635

636

637

638

639

640

641

642

643

644

645

646

647

648

649

650

651

ical Programming Computation 10, 187-223. URL: https://doi.org/10.1007/s12532-017-0127-0, doi:10.1007/s12532-017-0127-0.

Nikolić, D.D., 2016. Dae tools: equation-based object-oriented modelling, simulation and optimisation software. PeerJ Computer Science 2, e54. URL: https://doi.org/10.7717/peerj-cs .54, doi:10.7717/ peerj-cs.54.

Romeijn, H.E., Ahuja, R.K., Dempsey, J.F., Kumar, A., 2006. A new linear programming approach to radiation therapy treatment planning problems. Operations Research 54, 201-216. URL: https://doi.org/10.1287/opre.1050.0261, doi:10.1287/opre.1050.0261, arXiv:https://doi.org/10.1287/opre.1050.0261.

Rossum, G., 1995. Python Reference Manual. Technical Report. Amsterdam, The Netherlands, The Netherlands.

Silva, J.C., Bennett, L., Papageorgiou, L.G., Tsoka, S., 2016. A mathematical programming approach for sequential clustering of dynamic networks. The European Physical Journal B 89, 39. URL: https: //doi.org/10.1140/epjb/e2015-60656-5, doi:10.1140/epjb/e2015-60656-5.

Sitek, P., Wikarek, J., 2015. A hybrid framework for the modelling and optimisation of decision problems in sustainable supply chain management. International Journal of Production Research 53, 6611-6628. URL: http://dx.doi.org/10.1080/00207543.2015.1005762, doi:10.1080/00207543.2015.1005762, arXiv:http://dx.doi.org/10.1080/00207543.2015.1005762.

Tanveer, M., 2015. Robust and sparse linear programming twin support vector machines. Cognitive Computation 7, 137-149. URL: https://doi.org/10.1007/s12559-014-9278-8, doi:10.1007/ s12559-014-9278-8.

Triantafyllidis, C.P., Koppelaar, R.H., Wang, X., van Dam, K.H., Shah, N., 2018. An integrated optimisation platform for sustainable resource and infrastructure planning. Environmental Modelling Software 101, 146 - 168. URL: http://www. sciencedirect.com/science/article/pii/S1364815217301391, doi:https: //doi.org/10.1016/j.envsoft.2017.11.034.

Williams, H.P., 1999. Model Building in Mathematical Programming, 4th Edition. 4 ed., Wiley. URL: http://www . amazon.com/exec/obidos/redirect?tag=citeulike07-20\&path=ASIN/0471997889.

49 Yang, L., Liu, S., Tsoka, S., Papageorgiou, L.G., 2016. Mathematical programming for piecewise linear regression analysis. Expert Systems with Applications 44, 156-167. URL: http://dx.doi.org/10.1016/ j.eswa.2015.08.034, doi:10.1016/j.eswa.2015.08.034. 